\title{
A survey exploring characteristics of older people attending lunch cubs in South-West of England
}

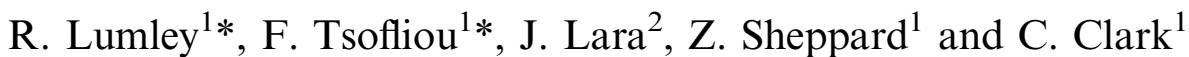 \\ ${ }^{1}$ Department of Human Sciences and Public Health, Faculty of Health and Social Sciences, Bournemouth University, \\ Bournemouth BH1 3LT, UK and ${ }^{2}$ Department of Applied Sciences, Faculty of Health and Life Sciences, Northumbria \\ University, Newcastle upon Tyne NE1 8ST, UK
}

Lunch Clubs (LCs) have been long considered a valuable community-based service to promote health and wellbeing of older people ${ }^{(1)}$, however people attending LCs are poorly characterised. The aim of this study was to explore the characteristics of those attending lunch clubs in South-West England and the reasons for attendance. Body weight $(\mathrm{kg})$, height $(\mathrm{m})$, hand-grip strength $(\mathrm{kg})$ and waist circumference (WC) $(\mathrm{cm})$ were measured by a trained observer following standard protocols. The length of attendance to LC, transport utilised and distance travelled to the LC was also explored. Participants reported self-rated health status, self-rated physical activity and reasons of attendance via a Likert scale.

Forty older individuals from 5 LCs completed the survey during the day of their LC visit. Characteristics are shown in the table below.

\begin{tabular}{|c|c|c|c|}
\hline & Men $(n=18)$ & Women $(n=22)$ & All subjects $(n=40)$ \\
\hline & \multicolumn{3}{|c|}{ Mean (Standard deviation) } \\
\hline Age (years) & 82 (SD 7) & 83 (SD 9) & 82 (SD 8) \\
\hline BMI $\left(\mathrm{kg} / \mathrm{m}^{2}\right)$ & $30(\mathrm{SD} 5)$ & $28(\mathrm{SD} 4)$ & 27 (SD 4) \\
\hline Waist circumference $(\mathrm{cm})$ & 104 (SD 12) & 97 (SD 12) & $100(\mathrm{SD} \mathrm{13})$ \\
\hline Grip strength (kg) & $23(\mathrm{SD} 5)$ & 14 (SD 5) & 18 (SD 6) \\
\hline
\end{tabular}

Overall, participants were older adults (65\% over 75 years old), overweight, abdominally obese with low hand-grip strength. Self-rated current health was rated as 'good to very good' by $68 \%$ of participants; $60 \%$ reported their current health being the same as the previous year, with $23 \%$ reporting an improvement and $18 \%$ declaring worsened health in relation with the previous year. Current self-reported physical activity was rated as 'moderate' by $63 \%$; low physical activity was reported by $28 \%$ of participants. Only $3 \%$ reported an increase in physical activity levels on the previous year with the remainder reporting levels "about the same" or lower (85\% and $12.5 \%$ respectively). Most $(65 \%)$ participants lived alone being either widowed $(68 \%)$, single $(8 \%)$ or divorced $(2 \%)$. None of them lived in a care home nor lived solely on benefits; $50 \%$ receive state pension. Word of mouth was the most popular $(70 \%)$ route to find out about the LC. $63 \%$ of participants lived within half a mile ratio from the LC; $27 \%$ were travelling over a mile to attend a LC. The most popular means of access to lunch club were getting a lift with a friend or walking to the lunch club (35\% and $35 \%$ of participants respectively). Overall, over $73 \%$ of participants rated that 'meeting friends' ( $92 \%$ ); 'to eat out' $(78 \%)$, 'to have a hot meal' $(75 \%)$, and 'for a home cooked style meal' $(73 \%)$ were important reasons to attend lunch clubs. Other factors such as 'not having to cook', 'affordability of a meal' and 'the additional activities (bingo, raffles and talks)' were rated as very important or important by only $45 \%, 40 \%$ and $18 \%$ of participants, respectively.

Older adults attending LCs in the South West of England reported good self-rated health, nonetheless they are characterised by high rates of overweight and obesity, abdominal obesity and low muscle strength placing them at risk of sarcopenia and sarcopenic obesity $^{(2)}$. These findings suggest the need for lifestyle interventions targeting these health problems.

1. Dwyer P \& Hardill I (2011) Ageing Soc 31, 243-264.

2. Prado CM, Wells JC, Smith SR et al. (2012) Clin Nutr 31, 583-601.

*Joint first authors 